\title{
A Rare Case of Hepatocellular Carcinoma Accompanied by Metastasis of a Cervical Lymph Node
}

\author{
Kiyoyuki Kobayashi ${ }^{1}$, Takashi Himoto ${ }^{2}$, Joji Tani ${ }^{1}$, Hisaaki Miyoshi ${ }^{1}$, Hirohito Yoneyama ${ }^{1}$, \\ Akihiro Deguchi ${ }^{1}$, Kazutaka Kurokohchi ${ }^{1}$, Hirohito Mori ${ }^{1}$, Hiroshi Hoshikawa ${ }^{3}$, \\ Masaki Ueno ${ }^{4}$ and Tsutomu Masaki ${ }^{1}$
}

\begin{abstract}
Metastasis to lymph nodes in patients with hepatocellular carcinoma (HCC) is generally observed to occur in regional chains of involvement. We encountered a HCC patient who had hepatitis C virus-related liver cirrhosis and portal vein tumor thrombosis, accompanied by metastasis to only a single cervical lymph node, skipping the common intermediate routes of involvement. It is noteworthy, so as not to miss the clinical diagnosis, to remark that metastasis to the cervical lymph node is rarely observed in patients with HCC, and that the metastasis can skip to the regional lymph nodes.
\end{abstract}

Key words: hepatocellular carcinoma, cervical lymph node, skip metastasis

(Intern Med 51: 381-385, 2012)

(DOI: 10.2169/internalmedicine.51.6764)

\section{Introduction}

Hepatocellular carcinoma (HCC) is one of the most common cancers worldwide; in general it arises from a precursor condition including chronic hepatitis or liver cirrhosis (1). Extrahepatic metastases of HCC are found in the lung, brain, adrenal gland, bones, lymph nodes and so on (2-5). The incidence of lymphatic metastasis of HCC is far lower than that of hematogeneous metastasis (2-4). Lymphatic metastasis usually occurs in regional chains; the so-called "skip metastasis" to the distal nodes seems to be rare (2-4). Here, we report an atypical case of $\mathrm{HCC}$ with metastasis of a cervical lymph node skipping the regional, mediastinal and supraclavicular lymph nodes.

\section{Case Report}

A 64-year-old Japanese man was referred to the Hospital of Kagawa University School of Medicine for detailed examinations of an enlarged cervical lymph node. He had a history of liver dysfunction at his health check-up 3 years prior. He had never received a blood transfusion, and did not take any medications, nor was he an alcohol abuser.

Physical examination on admission revealed no conjunctival anemia and no jaundice. He had no abnormal cardiopulmonary findings. The abdomen was soft and flat. The liver margin was palpated 3 fingerbreaths below the right costal margin. Pretibial edema was not found. No abnormal neurologic findings were observed. A left cervical lymph node was enlarged to approximately $3 \mathrm{~cm}$ in diameter, and felt elastic and firm on palpation, while supraclavicular, axillary and inguinal lymph nodes were not palpable.

Laboratory data on admission were as follows: his peripheral blood did not reveal thrombocytopenia (platelet: $16 \times 10^{4}$ cells $/ \mu \mathrm{L}$ ). Direct bilirubin (D-Bil), albumin (Alb) and prothrombin time (PT) were $0.6 \mathrm{mg} / \mathrm{dL}, 4.0 \mathrm{mg} / \mathrm{dL}$ and $97 \%$, respectively, suggesting a favorable hepatic reserve. However, the indocyanine green retention rate at $15 \mathrm{~min}$ (ICGR 15 ) was severely impaired (ICGR $15: 31.7 \%)$. Moreover, his blood chemistry indicated liver dysfunction with elevated levels of aspartate aminotransferase (AST) at $139 \mathrm{IU} / \mathrm{L}$,

\footnotetext{
${ }^{1}$ Department of Gastroenterology and Neurology, Kagawa University School of Medicine, Japan, ${ }^{2}$ Department of Integrated Medicine, Kagawa University School of Medicine, Japan, ${ }^{3}$ Department of Otolaryngology, Kagawa University School of Medicine, Japan and ${ }^{4}$ Department of Pathology and Host Defense, Kagawa University School of Medicine, Japan

Received for publication October 18, 2011; Accepted for publication November 3, 2011

Correspondence to Dr. Takashi Himoto, thimoto@med.kagawa-u.ac.jp
} 
alanine aminotransferase (ALT) at $111 \mathrm{IU} / \mathrm{L}$, lactate dehydrogenase $(\mathrm{LDH})$ at $354 \mathrm{U} / \mathrm{L}$ (normal: 100-220 U/L), gamma-glutamyl transpeptidase $(\gamma-\mathrm{GTP})$ at $175 \mathrm{U} / \mathrm{L}$ (normal: $<60 \mathrm{U} / \mathrm{L}$ ) and alkaline phosphatase (ALP) at $893 \mathrm{U} / \mathrm{L}$ (normal: 100-340 U/L). Viral serological tests revealed seronegativity for hepatitis B virus surface antigen ( $\mathrm{HBsAg}$ ) and seropositivity for the antibody to hepatitis $\mathrm{C}$ virus (anti$\mathrm{HCV}$ ). His load of HCV-RNA was $5.0 \log \mathrm{IU} / \mathrm{mL}$ by the HCV COBAS TaqMan HCV test.

Fine needle aspiration cytology of his cervical lymph node was performed and demonstrated groups of cells with enlarged hyperchromatic and central nuclei, prominent nucleoli, intranuclear inclusions and coarse granular cytoplasm, suggesting poorly differentiated cancer cells, although we did not determine the histological type (Fig. 1). He underwent positron emission tomography (PET) to search for a primary lesion. The test revealed dense uptakes of ${ }^{18} \mathrm{~F}$ -

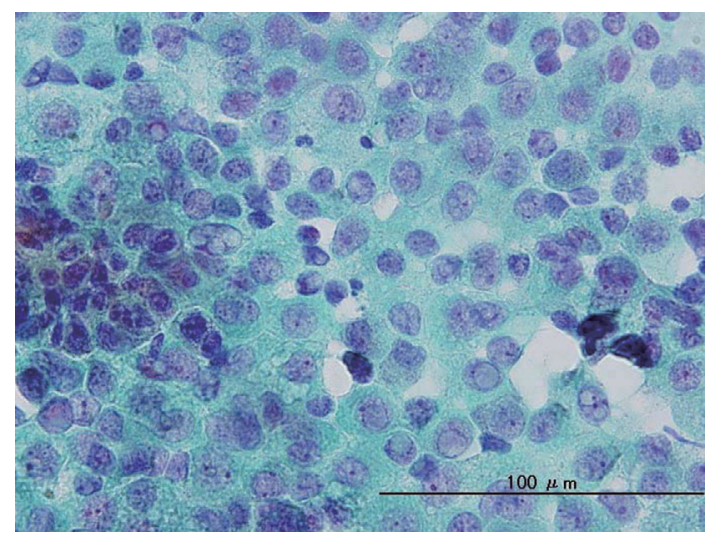

Figure 1. Fine needle aspiration cytology of a cervical lymph node showing undifferentiated carcinoma. (Papanicolau stain, original magnification $\times \mathbf{2 0 0}$ ) fluorodeoxyglucose $\left({ }^{18} \mathrm{~F}-\mathrm{FDG}\right)$ in the liver (Fig. 2a) as well as in a left cervical lymph node (Fig. 2b), suggesting metastasis to the cervical lymph node from the liver tumors. No other accumulations to imply lymph node metastases were found in the peritoneum, mediastinum or axilla. A dynamic computed tomography (CT) scan of the abdomen revealed multiple nodules which showed washout in the right lobe of the cirrhotic liver (Fig. 3a), accompanied by a small amount of ascites around the liver (Fig. 3b), during the portal dominant phase. Tumor thrombosis was also observed in the right branch of the portal vein (Fig. 3b). His tumor markers for hepatocellular carcinoma (HCC) including $\alpha$-fetoprotein (AFP), AFP-L3 fraction and plasma protein induced by vitamin $\mathrm{K}$ absence or antagonist-II (PIVKA-II) were all elevated [AFP: 164,878 ng/mL (normal <10 ng/mL), AFP-L3 82.8\% (normal <10\%), PIVKA-II: $65,400 \mathrm{mAU} / \mathrm{mL}$ (normal $<40$ $\mathrm{mAU} / \mathrm{mL})]$. On the basis of laboratory, radiological and pathological data, he was diagnosed with HCC accompanied by metastasis of a cervical lymph node.

He was treated with 3 courses of hepatic arterial infusion chemotherapy (HAIC) using 5-fluorouracil (5-FU) in combination with low-dose cisplatin (CDDP). However, his HCC was refractory to the chemotherapy and continued to progress. Thereafter, his hepatic reserve was severely impaired, resulting in deep jaundice and multiple ascites. He died of hepatic failure.

\section{Autopsy}

Macroscopic findings revealed that HCC occupied a large part of right lobe of the liver (Fig. 4a). The underlying liver disease was micronodular liver cirrhosis. The enlarged cervical lymph node was $42 \times 30 \mathrm{~mm}$ in size and encapsulated (Fig. 4b). No metastases to the regional, mediastinal and axillar lymph nodes were observed. Histologically, moderately

(a)

(b)

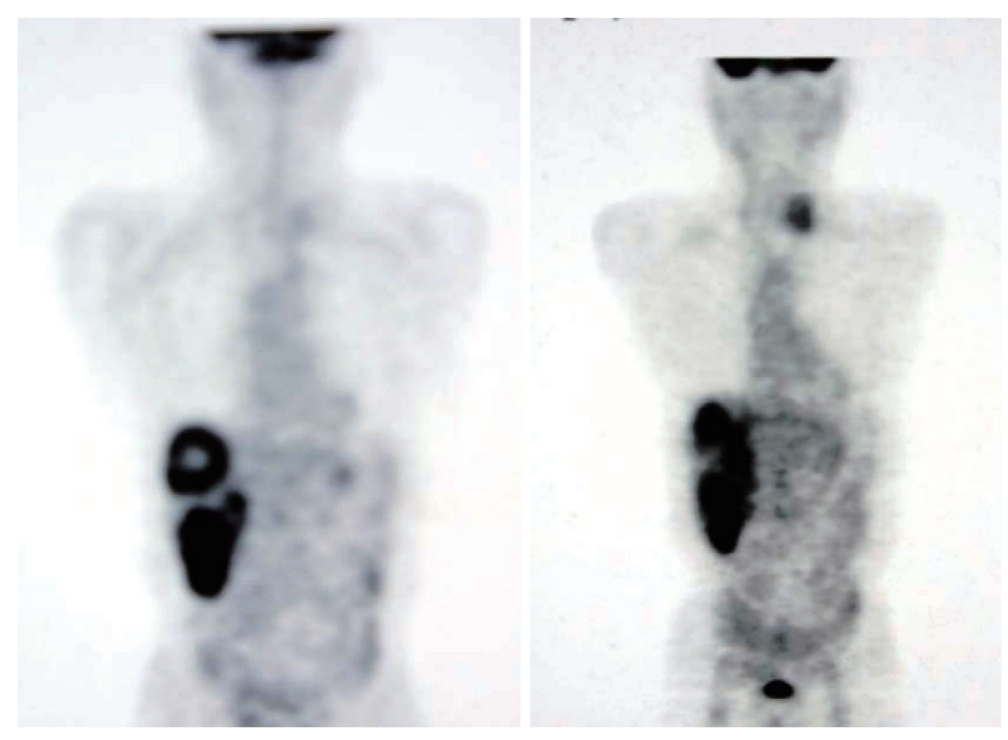

Figure 2. PET test showing uptakes of ${ }^{18}$ F-FDG into (a) the liver as well as (b) a cervical lymph node. 
(a)

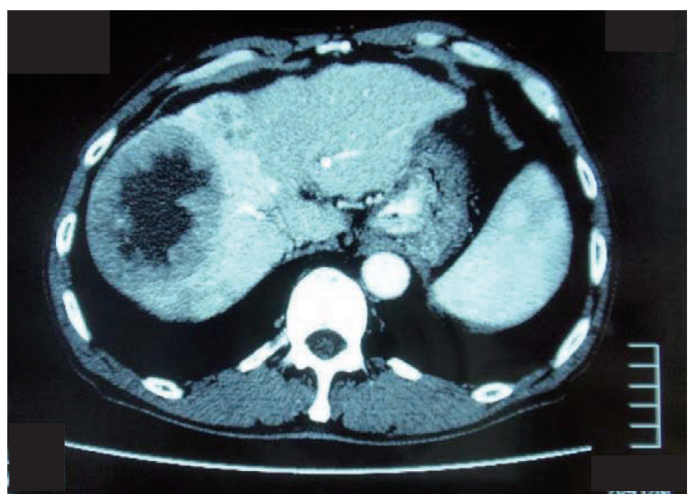

(b)

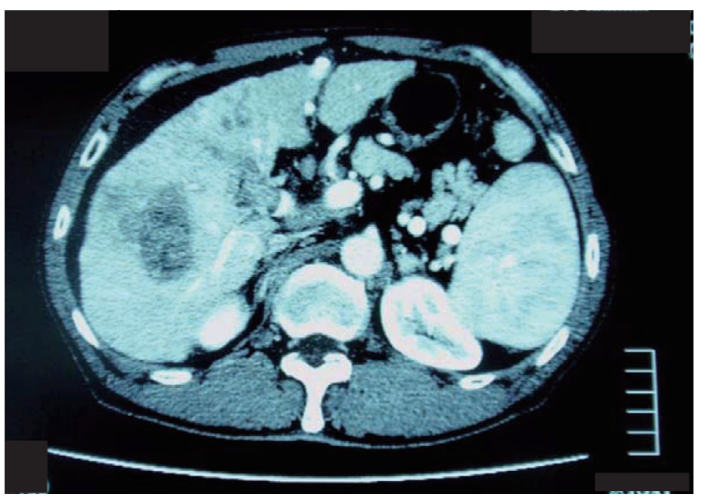

Figure 3. Dynamic CT scan showing (a) multiple liver nodules which were washed out and (b) portal vein tumor thrombus during the portal dominant phase.

(a)

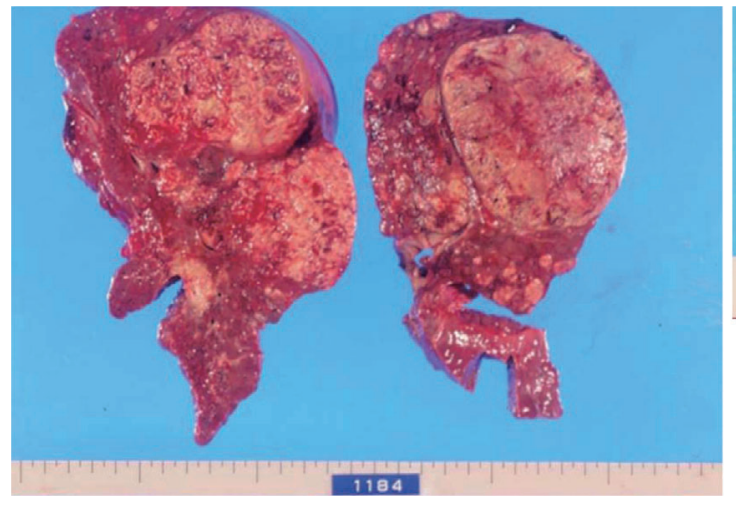

(b)

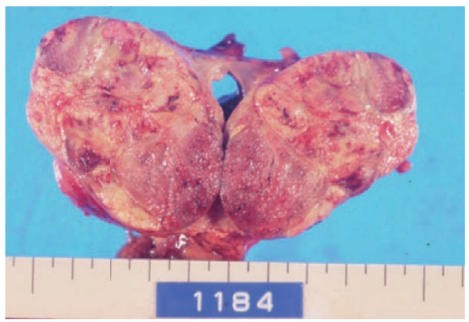

Figure 4. Gross appearance of (a) the liver tumors and (b) a cervical lymph node. Liver tumors occupied most of the right lobe of the liver. The enlarged cervical lymph node was encapsulated.

(a)

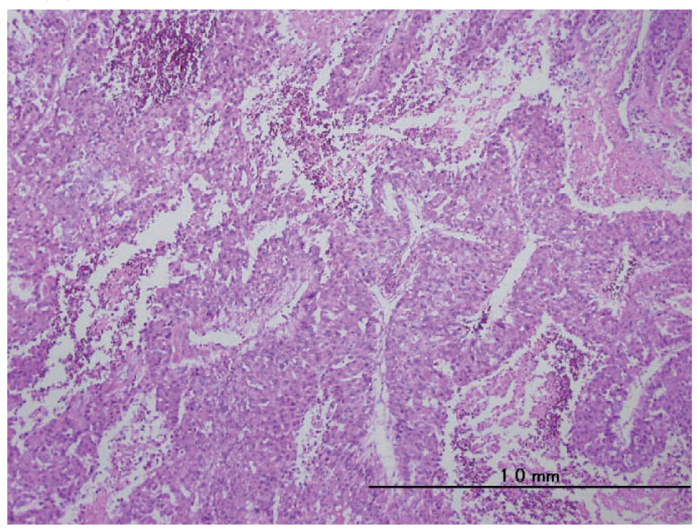

(b)

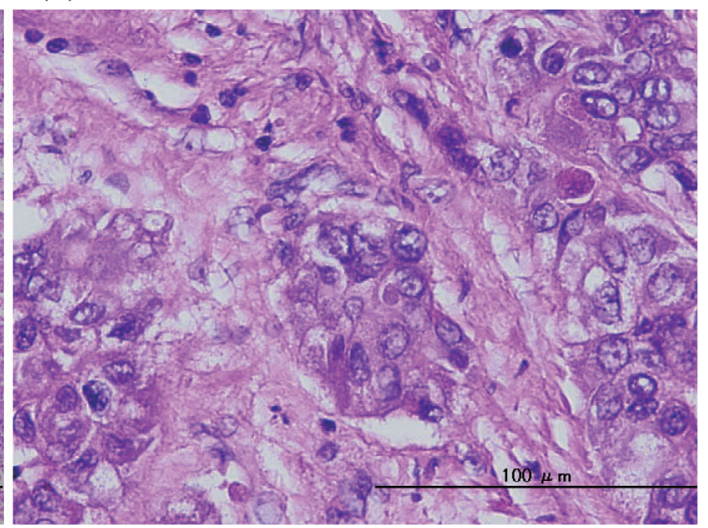

Figure 5. Histological appearances of liver tumor consisting of moderately to poorly differentiated HCC with trabecular arrangements (Hematoxylin and Eosin stain, original magnification (a) $\times 100$, (b) $\times 400$ ).

to poorly differentiated HCC was demonstrated in the liver tumor, which was accompanied with a trabecular arrangement (Fig. 5). The histological appearance in the cervical lymph node was almost the same as that in the liver tumor. Therefore, autopsy findings confirmed the diagnosis of HCC with metastasis to the cervical lymph node. The clinical stage of this patient corresponded to stage $4 \mathrm{~b}$ because of distal lymph node metastasis in accordance with the TNM classification of HCC (6). 


\section{Discussion}

Previous autopsy studies have found lymph node metastasis in approximately $25 \%$ to $40 \%$ of patients with HCC (2-4). Lymphatic spread of HCC ordinarily occurs to the hepatic hilar, peripancreatic and perigastric lymph nodes (2-4). However, mediastinal (7), axillary (8), subraclavicular (9), and cervical (10) lymph nodes are unusually infiltrated. Abe and colleagues (11) revealed the relationship between the type of primary lesions and the patterns of lymph node metastasis. According to their report, primary lesions can be classified into 3 types: massive HCC with portal vein tumor thrombus (type A), multiple tumors of small nodules without capsule (type B) and encapusulated nodular type (type C). Type A and B are more extensively accompanied by lymph node metastasis than type C. It was noted that HCC patients with type A and B had underlying liver disease of liver cirrhosis. The present patient's lesions were classified as type A, and he indeed had liver cirrhosis as the underlying liver disease. On the other hand, Watanabe and colleagues (3) documented that lymph node metastasis was frequently associated with poorly differentiated HCC. The present case was also classified into moderately to poorly differentiated HCC. The prognosis of HCC patients with lymph node metastases generally tends to be poor $(3,11)$.

It is of interest that the relationship between the location of HCC and the pattern of lymph node metastasis was described in a previous study (3). In particular, cervical lymph node metastasis appeared in 4 of $35(11.4 \%)$ HCC patients whose tumors were in the upper right lobe of the liver, but, in none of the $12 \mathrm{HCC}$ patients whose tumors were in the lower right lobe. In the present case, the main tumors were largely located in the upper right lobe of the liver, in accordance with the previously documented pattern.

The hepatic lymphatic drainage system has been well established. Most lymph flow towards the hepatic hilum runs through the hepatoduodenal ligaments. On the other hand, a small amount of flow communicates with the diaphragmatic and intrathoracic lymphatic system through the bilateral triangular ligaments (12). In the present patient, the latter pathway might have been associated with the metastasis of the cervical lymph node. It is of interest that this patient had no metastasis of regional, mediastinal and axillary lymph nodes; in other words, he had "skip" metastasis (13). Increased lymph production is often observed in patients with HCC, because most patients with HCC have underlying chronic liver disease including chronic hepatitis or liver cirrhosis (10). The complex bypasses of lymphatic flow due to lymphatic occlusion may account for the skip metastasis of lymph nodes. The present case had liver cirrhosis as an underlying liver disease. Moreover, his HCC was associated with portal vein tumor thrombosis. These factors may contribute to the occurrence of the skip metastasis in this case.

Surgical resection (14) and radiation (15) have been con- sidered treatments for recurrent lymph node metastasis of HCC after partial hepatectomy if the lymph node metastasis is solitary. In this case, HAIC was selected as the treatment for HCC in accordance with consensus-based treatment algorithm for HCC proposed by the Japan Society of Hepatology (16), because our patient had advanced HCC with major portal vein tumor thrombosis. Wada and colleagues (17) reported the efficacy of HAIC using 5-FU and interferon in patients with HCC accompanied with portal vein tumor thrombus and lymph node metastasis. Unfortunately, the present patient was refractory to the chemotherapy.

In summary, the metastasis of a cervical lymph node alone is rarely observed in patients with HCC. Clinicians should be aware of the possibility of skip metastasis accompanying HCC.

The authors state that they have no Conflict of Interest (COI).

\section{References}

1. Scherman M. Hepatocellular carcinoma: epidemiology, surveillance, and diagnosis. Semin Liver Dis 30: 3-16, 2010.

2. Yuki K, Hirohashi S, Sakamoto M, et al. Growth and spread of hepatocellular carcinoma. A review of 240 consecutive cases. Cancer 66: 2174-2179, 1990.

3. Watanabe J, Nakashima O, Kojiro M. Clinicopathologic study on lymph node metastasis of hepatocellular carcinoma: a retrospective study of 660 consecutive autopsy cases. Jpn J Clin Oncol 24: 3741, 1994

4. Katyal S, Oliver JH 3rd, Peterson MS, et al. Extrahepatic metastases of hepatocellular carcinoma. Radiology 216: 698-703, 2000.

5. Ikai I, Arii S, Okazaki M, et al. Report of 17 th nationwide followup survey of primary liver cancer in Japan. Hepatol Res 37: 676691, 2007.

6. Greene FL, Page DL, Fleming ID, et al. AJCC Cancer Saging Manual. 6th edn. Springer, Chicago, 2002: 131-144.

7. Tanaka O, Kanematsu M, Kondo H, et al. Solitary mediastinal lymph node metastasis of hepatocellular carcinoma: MR imaging findings. Magnetic Resonance Imaging 23: 111-114, 2005.

8. Alison MR, Leiman G, Kew MC. Metastasis in an axillary lyph node in hepatocellular carcinoma: a case report. World J Gastroenterol 6: 770-772, 2000.

9. Selvasekaran R, Cherian JV, Venkataraman J. Metastasis of hepatocellular carcinoma to Virchow's node: Have the tumor cells gone astray. Hepatobiliary Pancreat Dis Int 6: 650-652, 2007.

10. Köklü S, Arhan M, Köksal A, et al. An unusual presentation of hepatocellular carcinoma. Int J Gastrointest Cancer 34: 63-65, 2003.

11. Abe T, Furuse J, Yoshino M, et al. Clinical characteristics of hepatocellular carcinoma with an extensive lymph node metastasis at diagnosis. Am J Clin Oncol 25: 318-323, 2002.

12. Magari S. Hepatic lymphatic system: structure and function. J Gastroenterol Hepatol 5: 82-93, 1990.

13. Uehara K, Hasegawa H, Ogiso $S$, et al. Skip lymph node metastases from a small hepatocellular carcinoma with difficulty in preoperative diagnosis. J Gastroenterol Hepatol 18: 345-349, 2003.

14. Uenishi T, Hirohashi $K$, Shuto $T$, et al. The clinical significance of lymph node metastases in patients with undergoing surgery for hepatocellular carcinoma. Surg Today 30: 892-895, 2000.

15. Park YJ, Lim DH, Paik SW, et al. Radiation therapy for abdominal luph node metastasis from hepatocellular carcinoma. J Gastroenterol 41: 1099-1106, 2006. 
Intern Med 51: 381-385, 2012 DOI: 10.2169/internalmedicine.51.6764

16. Arii S, Sata M, Sakamoto $M$, et al. Management of hepatocellular carcinoma: report of consensus meeting in the 45th annual meeting of the Japan Society of Hepatology (2009). Hepatol Res 40: 667-685, 2010.

17. Wada H, Nagano H, Doda T, et al. Complete remission of hepato- cellular carcinoma with portal vein tumor thrombus and lymph node metastases by arterial infusion of 5-fluorouracil and interferon- $\alpha$ combination therapy following hepatic resection. J Gastroenterol 42: 501-506, 2007.

(C) 2012 The Japanese Society of Internal Medicine http://www.naika.or.jp/imindex.html 